\title{
Design issues toward a cost effective physical layer for multiband OFDM (ECMA-368) in consumer products
}

Article

Published Version

Sherratt, R.S. (2006) Design issues toward a cost effective physical layer for multiband OFDM (ECMA-368) in consumer products. IEEE Transactions on Consumer Electronics, 52 (4). pp. 1179-1183. ISSN 0098-3063 doi:

https://doi.org/10.1109/TCE.2006.273131 Available at https://centaur.reading.ac.uk/15373/

It is advisable to refer to the publisher's version if you intend to cite from the work. See Guidance on citing.

To link to this article DOI: http://dx.doi.org/10.1109/TCE.2006.273131

Publisher statement: (c) 2006 IEEE. Personal use of this material is permitted. Permission from IEEE must be obtained for all other users, including reprinting/ republishing this material for advertising or promotional purposes, creating new collective works for resale or redistribution to servers or lists, or reuse of any copyrighted components of this work in other works.

All outputs in CentAUR are protected by Intellectual Property Rights law, including copyright law. Copyright and IPR is retained by the creators or other copyright holders. Terms and conditions for use of this material are defined in the End User Agreement. 


\section{www.reading.ac.uk/centaur}

\section{CentAUR}

Central Archive at the University of Reading

Reading's research outputs online 


\title{
Design Issues Toward a Cost Effective Physical Layer for Multiband OFDM (ECMA-368) in Consumer Products
}

\author{
R. Simon Sherratt, Senior Member, IEEE
}

\begin{abstract}
The creation of Wireless Personal Area Networks (WPANs) offers the Consumer Electronics industry a mechanism to truly unwire consumer products, leading to portability and ease of installation as never seen before. WPAN's can offer data-rates exceeding those that are required to convey high quality broadcast video, thus users can easily connect to high quality video for multimedia presentations in education, libraries, advertising, or have a wireless connection at home.

There have been many WPAN proposals, but this paper concentrates on ECMA-368 as this standard has the largest industrial and implementers' forum backing. With the aim to define and create cost effective consumer electronic equipment this paper discusses the technology behind ECMA368 physical layer, the design freedom availabilities, the required processing, buffer memory requirements and implementation considerations while concentrating on supporting all the offered data-rates ${ }^{l}$.
\end{abstract}

Index Terms - WiMedia, ECMA-368, specification, performance modeling, W-USB, UWB.

\section{INTRODUCTION}

After the US Federal Communications Commission (FCC) released frequency space for Ultra Wideband (UWB) transmission in 2002 [1], Ellis, Siwiak and Roberts published the initial requirements specification for UWB devices [2]. UWB offers high bandwidth wireless communications (order of $480 \mathrm{Mbit} / \mathrm{sec}$ to $1 \mathrm{Gbit} / \mathrm{sec})$ over a small distance $(<10$ meters). To offer such a large bandwidth, UWB uses the 3.110.6 GHz RF range in the USA (with other countries depending on local regulatory issues) and assumes stationary devices, i.e. a time-invariant propagation channel. Since the requirements document was published, many UWB proposals were made under IEEE 802.15.3a to converge on an agreed solution. However, 2 clear candidates quickly emerged, DSUWB [3] and MB-OFDM [4]. Many groups assessed the viability of the 2 proposals. In parallel with the standardization attempts, an industrial consortium termed the Multiband OFDM Alliance Special Interest Group (MBOASIG) forged ahead to standardize their UWB system based on MB-OFDM [4]. A key activity in MBOA development was that MB-OFDM has been selected by the Universal Serial Bus (USB) Implementers Forum [5] for the new Wireless-USB Physical (PHY) layer standard [6]. In 2005 MBOA was consumed into the new MiMedia Alliance. In September

\footnotetext{
${ }^{1}$ R. Simon Sherratt is with the Signal Processing Laboratory, School of Systems Engineering, The University of Reading, RG6 6AY, UK (e-mail: r.s.sherratt@reading.ac.uk).
}

Contributed Paper

Manuscript received September 11, 2006

0098 3063/06/\$20.00 @ 2006 IEEE
2004, the FCC issued a waver to operate MBOA [7] and on the 10th March 2005 the FCC gave the WiMedia Consortium approval to sell UWB products in the USA [8]. In December 2005 the WiMedia Alliance published the first version of their UWB PHY and Medium Access Control (MAC) standard termed ECMA-368 [9] which is based on the technology in the previous MBOA proposal [10] with only minor changes. The MAC/PHY interface standard ECMA-369 [11] was also proposed for the first time.

ECMA-368 has a huge potential for the Consumer Electronics market. It offers bandwidth large enough for highquality video broadcast over small wireless links. This feature will enable Consumer Devices to become truly wireless (for example a DVD player and display device can be connected wirelessly). For the Computer industry a Wireless-USB capable of full-rate USB 2.0 (53Mbyte/sec sustained) suitable for laptops and desktops alike will again enable connectivity. New features such as watching broadcast quality video over a broadcast-type wireless link will enable multimedia presentation for offices, airports, education, museums and advertising. Recently the Bluetooth-SIG announced their Bluetooth Protocol Adaptation Layers for use with ECMA368 thus offering fast Bluetooth over the same physical layer as Wireless-USB (yet to be fully standardized) [12].

To create a market acceptable ECMA-368 solution, the device must not only be standards conformant, but costeffective, low power, have high performance and capable of all the available coding rates so as to be as flexible and to have the same device in many products and services, thus lowering costs by reuse. This paper discusses design issues to the digital baseband that designers must be aware of at the system specification stage in order to create an effective solution.

\section{ECMA-368 PHY INTRODUCTION}

ECMA-368 [9] defines the Multiband OFDM based UWB standard by the WiMedia Consortium based on the initial proposal by Batra et al [4]. ECMA-368 defines the PHY and MAC interface. In this paper, the concern is to the PHY layer as this layer defines the transport data-rate ability, the performance and is the major contributor to cost, chip-size and electrical power.

For each MAC frame required to be transmitted, a PLCP (Packet Layer Convergence Protocol) Packet Data Unit (PPDU) must be formed consisting of 3 parts, the PLCP Preamble (containing the Packet/Frame Sync and the Channel Estimation sequence), the PLCP Header and the PLCP Service Data Unit (PSDU) as per Fig. 1. The contents of the PLCP 


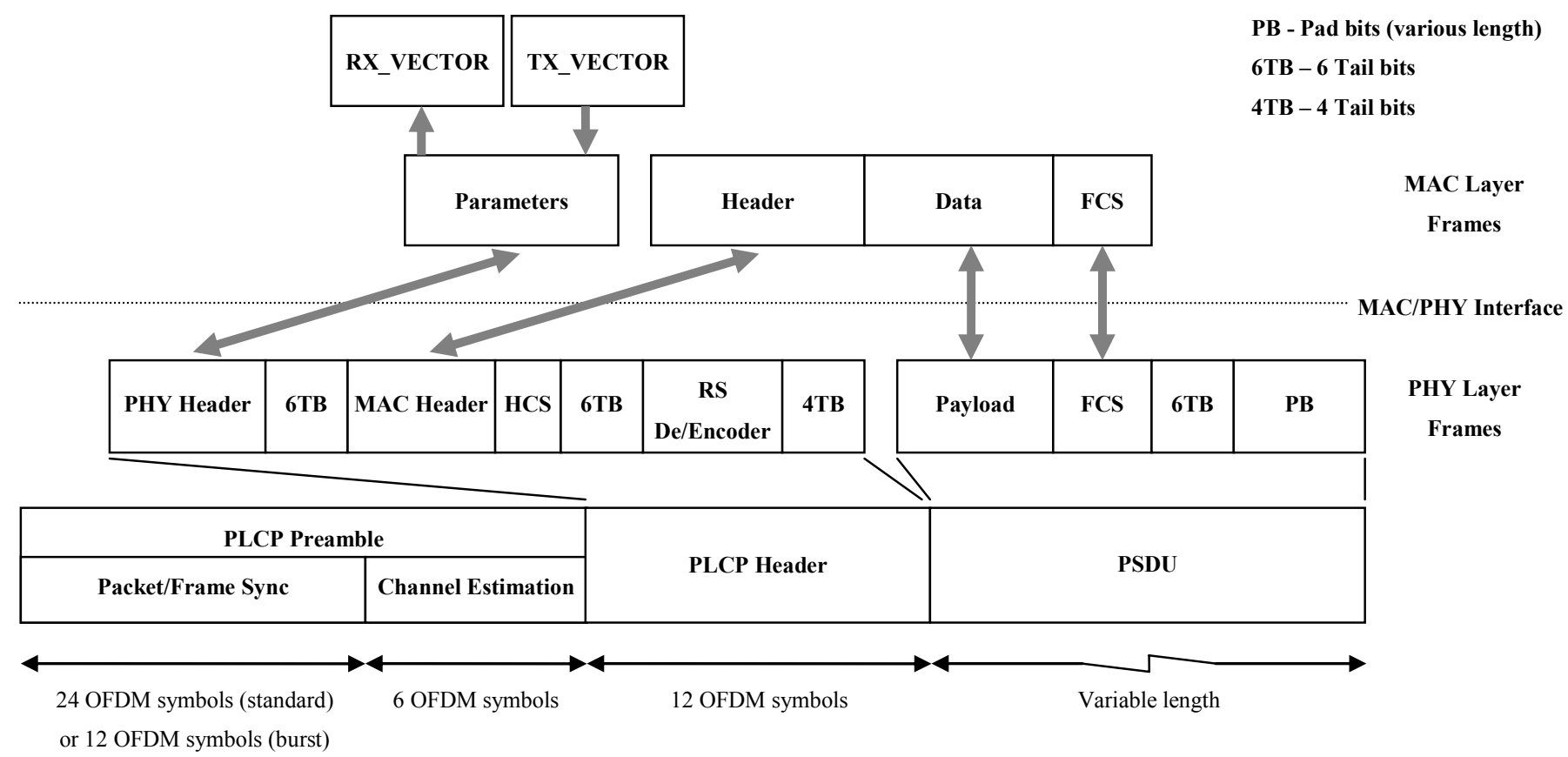

Fig. 1. PLCP and PSDU arrangement for ECMA-368, PLCP Preamble may be stored in ROM whereas the PLCP Header and PSDU contain a sequence of OFDM symbols.

Preamble are known a-priori and can be stored in ROM, whereas the PLCP Header and PSDU contain coded data modulated into OFDM symbols.

A 128-pt IFFT is used to create the ODFM symbols at a fixed rate of $242.42 \mathrm{~ns}$ irrespective of requested data-rate. Each OFDM symbol has a 37-sample zero value guard interval appended to the end of the symbol giving a total OFDM symbol time of $312.5 \mathrm{~ns}$. Each OFDM symbol is made from 100 data sub-carriers, 12 pilot sub-carriers, 10 guard sub-carriers (copy of the outer 5 data sub-carriers to each symbol) and 6 null-valued pilots (5 high frequency and the DC term).

ECMA-368 defines 5 Band Groups (BG1-BG5) using $3 \times 528 \mathrm{MHz}$ channels in Band Groups 1-4, and 2 channels in BG5. A Time-Frequency Code (TFC) is given to the PHY from the MAC to define the hopping sequence across the selected BG while hopping is only performed in BG1-4.

The instantaneous (not sustained) data-rates available to the MAC transport are 53.3, 80, 106.7, 160, 200, 320, 400 and $480 \mathrm{Mbit} / \mathrm{sec}$ formed by a mixture of various puncturing, time and frequency spreading (diversity). This paper will present the sustainable data rates in section IV. For conformance a device must at least support the 53.3, 106.7 and $200 \mathrm{Mbit} / \mathrm{sec}$ rate using BG1.

For data rates of $200 \mathrm{Mbit} / \mathrm{sec}$ and below, a Time-Domain Spreading (TDS) scheme is employed to send an OFDM symbol on a carrier in 1 channel of the selected BG and then to send the same OFDM symbol (with the same polarity or inverted polarity) on the next carrier in the same $\mathrm{BG}$ as defined by the TFC.

For data rates of 53.3, $80 \mathrm{Mbit} / \mathrm{sec}$ (and the PLCP Header), a Frequency-Domain Spreading (FDS) scheme is employed to QPSK (Quadrature Phase Shift Keying) map each bit-pair into a complex-value and then copy onto 2 IFFT sub-carriers thus improve performance in Frequency Selective Fading. This is achieved by placing the complex-value onto the same positive and negative (i.e. complex conjugate) frequency input, resulting in real-only time domain output.

For data rates above $320 \mathrm{Mbit} / \mathrm{sec}$ Dual Carrier Modulation (DCM) is used instead of QPSK. As these data rates do not have time or frequency spreading then DCM is used to achieve a lower level of intra-symbol frequency diversity as the interleaver output bits are grouped into 4-bits and these bits are mapped into 2 indexes applied into the IFFT. This scheme offers the road-map to near Gbit/sec by replacing the DCM with conventional 16-QAM using 4-bits per 1 symbol.

\section{A. PLCP Preamble}

As with many wireless systems, a preamble is sent to aid receiver synchronization. ECMA-368 defines 2 packet/frame preambles, being the standard preamble of 24 a-priori timedomain sequences sent instead of an OFDM symbol, or a burst preamble of 12 a-priori time-domain sequences. Where the data to be sent spans multiple PPDU's, the burst mode may be selected to reduce the preamble overhead, of note is that the first PPDU always uses the standard preamble sequence while indicating if the next PPDU uses the standard or burst preamble. The burst preamble scheme is ideal for data-streaming as the receiver can maintain lock between successive PPDU's.

Following the 24 or 12 packet/frame sequence, 6 identical value a-priori OFDM Channel Estimation symbols are sent. 
TABLE I

CODING APPLIED TO PSDU DEPENDANT ON REQUESTED DATA-RATE

\begin{tabular}{cccccc}
\hline \hline $\begin{array}{c}\text { Rate } \\
\text { (Mbit/sec) }\end{array}$ & TDS & Code-rate & Modulation & $\begin{array}{c}\text { FFT } \\
\text { type }\end{array}$ & $\begin{array}{c}\text { Interleaver } \\
\text { block size }\end{array}$ \\
\hline $\mathbf{5 3 . 3}$ & YES & $1 / 3$ & QPSK & conjugate & 300 \\
$\mathbf{8 0}$ & YES & $1 / 2$ & QPSK & conjugate & 300 \\
$\mathbf{1 0 6 . 7}$ & YES & $1 / 3$ & QPSK & Non-conjugate & 600 \\
$\mathbf{1 6 0}$ & YES & $1 / 2$ & QPSK & Non-conjugate & 600 \\
$\mathbf{2 0 0}$ & YES & $5 / 8$ & QPSK & Non-conjugate & 600 \\
$\mathbf{3 2 0}$ & NO & $1 / 2$ & DCM & Non-conjugate & 1200 \\
$\mathbf{4 0 0}$ & NO & $5 / 8$ & DCM & Non-conjugate & 1200 \\
$\mathbf{4 8 0}$ & NO & $3 / 4$ & DCM & Non-conjugate & 1200 \\
\hline
\end{tabular}

TABLE II

AVAILABLE PSDU THROUGHPUT FOR PSDU LENGTH OF 4095 OCTETS

\begin{tabular}{|c|c|c|c|c|c|}
\hline $\begin{array}{c}\text { Rate } \\
\text { (Mbit/sec) }\end{array}$ & $\begin{array}{l}\text { PLCP Preamble } \\
\text { Type, time }\end{array}$ & $\begin{array}{c}\text { Number of } \\
\text { transmission } \\
\text { OFDM } \\
\text { Symbols } \\
\end{array}$ & $\begin{array}{c}\text { PSDU } \\
\text { Transmission } \\
\text { time } \\
\text { (us) } \\
\end{array}$ & $\begin{array}{c}\text { PPDU } \\
\text { Transmission } \\
\text { time } \\
\text { (us) } \\
\end{array}$ & $\begin{array}{c}\text { Available } \\
\text { Throughput } \\
\text { per frame } \\
\text { (Mbit/sec) }\end{array}$ \\
\hline \multirow[t]{2}{*}{53.3} & Standard, 9.375us & 1968 & 615 & 628.125 & 52.155 \\
\hline & Burst, 5.625us & 1968 & 615 & 624.375 & 52.468 \\
\hline \multirow[t]{2}{*}{80} & Standard, 9.375 us & 1314 & 410.63 & 423.75 & 77.31 \\
\hline & Burst, 5.625us & 1314 & 410.63 & 420 & 78 \\
\hline \multirow[t]{2}{*}{106.7} & Standard, 9.375 us & 984 & 307.5 & 320.625 & 102.18 \\
\hline & Burst, 5.625us & 984 & 307.5 & 316.875 & 103.38 \\
\hline \multirow[t]{2}{*}{160} & Standard, 9.375 us & 660 & 206.25 & 219.375 & 149.33 \\
\hline & Burst, 5.625us & 660 & 206.25 & 215.625 & 151.93 \\
\hline \multirow[t]{2}{*}{200} & Standard, 9.375 us & 528 & 165 & 178.125 & 183.92 \\
\hline & Burst, 5.625us & 528 & 165 & 174.375 & 187.87 \\
\hline \multirow[t]{2}{*}{320} & Standard, 9.375 us & 330 & 103.13 & 116.25 & 281.81 \\
\hline & Burst, 5.625us & 330 & 103.13 & 112.5 & 291.2 \\
\hline \multirow[t]{2}{*}{400} & Standard, 9.375 us & 264 & 82.5 & 95.625 & 342.59 \\
\hline & Burst, 5.625us & 264 & 82.5 & 91.875 & 356.57 \\
\hline \multirow[t]{2}{*}{480} & Standard, 9.375 us & 222 & 69.375 & 82.5 & 397.09 \\
\hline & Burst, 5.625us & 222 & 69.375 & 78.75 & 416 \\
\hline
\end{tabular}

TABLE III

REQUIRED INTERMEDIATE TRANSCEIVER MEMORY BUFFERING ASSUMING SERIAL BIT-ENCODING AND TRANSMITTER QUEUE OF 1 OFDM SYMBOL

\begin{tabular}{ccc}
\hline Rate & Size (octets) & Type \\
(Mbit/sec) & & \\
\hline TX/RX PLCP Preamble & 896 & 7 base TF sequences at 128 octets each \\
TX/RX MAC Interface Buffer & $16+4095+4$ & Channel estimation sequence \\
TX PLCP & $17+23$ & TX/RX VECTOR, PSDU and MAC FCS \\
Specific & $17+23$ & RS Encoder input \\
TX PLCP/PSDU & 150 & RS Encoder output \\
Shared operations & $2 * 128$ & Interleaver input buffer \\
& $2 * 128$ & QPSK/DCM output and IFFT input \\
RX PLCP & $2 * 128$ & IFFT current output \\
Specific & $17+23$ & IFFT output queue and spread \\
RX PLCP/PSDU & $17+23$ & RS Decoder input \\
Shared operations & $2 * 2 * 128$ & RS Decoder output \\
& $2 * 128$ & Main and de-spread buffers \\
& $2 * 100$ & FFT output buffer \\
& 1200 & Equalizer output buffer \\
Total & $3 *(4095+4+1)$ & De-interleaver output buffer \\
& $(4095+4+1) / 8$ & Soft Convolutional decoder trace-back memory \\
& $\mathbf{2 1 1 9 8}$ & Total baseband memory (octets) assuming serial bit \\
& & encoding and 1 OFDM output queue.
\end{tabular}

\section{Receiver Parameters Extraction}

In the radio channel frames are often corrupted and in ECMA-368 a mechanism exists to check if the received PLCP Header is valid (the MAC Header and data are checked at the MAC level by the MAC FCS - thus no PSDU checking is performed at the PHY). As the parameters to decode the
ECMA-368 is based on the older MBOA PHY standard [10]. In this standard, 6 tail bits existed to fully pin the $1 / 3$ rate constraint length 7 convolutional coder used in the PLCP. However, ECMA-368 uses a RS encoder with 48 parity bits leaving only space for 4 bits for PLCP Header state pinning. As a consequence the receiver convolutional decoder is not fully pinned and the receiver must select the best metric to 
trace-back from as opposed to being fully pinned at state 0 . In this case the 4 tail bits result in the decoder examining states $0,1,2$ and 3 to see which the best metric is and where to trace-back from. Also, as the constraint length is 7, but only 4 tail bits exist, then an extra $2 * 3$ 'dummy' symbols must be inserted at the end of the PLCP Header so that the decoder can flush the received bits out in a constrained fashion. It is possible to use the first 6 symbols of the PSDU, but this assumes that in the receiver the PLCP Header buffer memory is placed just before the PSDU buffer memory and determinate operation can be guaranteed as is not always the case in modern optimizing compilers.

\section{Receiver RS Decoder}

The PLCP Header contains all the required information to decode the PSDU and is coded at a fixed 1/3 code with FDS and TDS irrespective of PSDU rate.

The implementation of the RS encoder in the transmitter PLCP Header is mandatory, but since the RS encoding is a systematic code, the implementation of the RS decoder in the receiver is optional and relates to the required performance vs. available processing, power and physical die size. To make a decision to include the RS decoder, Fig. 2. depicts the PLCP Header Packet Error Rate (PER) performance of ECMA-368 conformant simulation using Forester's Channel Model 4 (CM4) (extreme non-line of sight) [13] with and without implementing the RS decoder in the receiver. As can be seen and as expected the PER performance is better with the RS decoder, but at the $\mathrm{PER}=8 \%$ conformance figure only $0.5 \mathrm{~dB}$ in performance has been gained, similar figures are obtained with the other Forester channels. Also it can be seen that when the PER is $8 \%$ with the RS decoder then the absence of the RS decoder only gives a PER $12 \%$. This result gives the designer freedom to select performance vs. power/size tradeoffs. With a similar thesis to above, Fig. 3. depicts the PLCP Header BitError Rate (BER) performance of ECMA-368 conformant simulation using CM-4. A performance loss of 0.3-0.4 dB can be seen which is as expected in the same order as the above.

\section{Time Domain De-Spreading}

For the $200 \mathrm{Mbit} / \mathrm{sec}$ rates and below (and the PLCP Header), TDS is used. The reception of each OFDM symbol within the pair of spread symbols would require their own FFT, channel estimation and equalization processes. The 2 equalizer outputs would need to be processed to see which symbol (or each sub-carrier) would be selected for QPSK demapping. However, a minimal receiver may be created to only decode 1 of the 2 packets for reception caused by the spreading. In this case, although the RF and ADC are operating at the same rate as if receiving 2 symbols, only 1 receiver FFT, channel estimator and equalizer process are required over the 2 possible symbols, this halving clock rates or only needing 1 FFT, channel estimation and equalization process. Considering the large impact an FFT operation and equalization operation has on an OFDM based receiver then

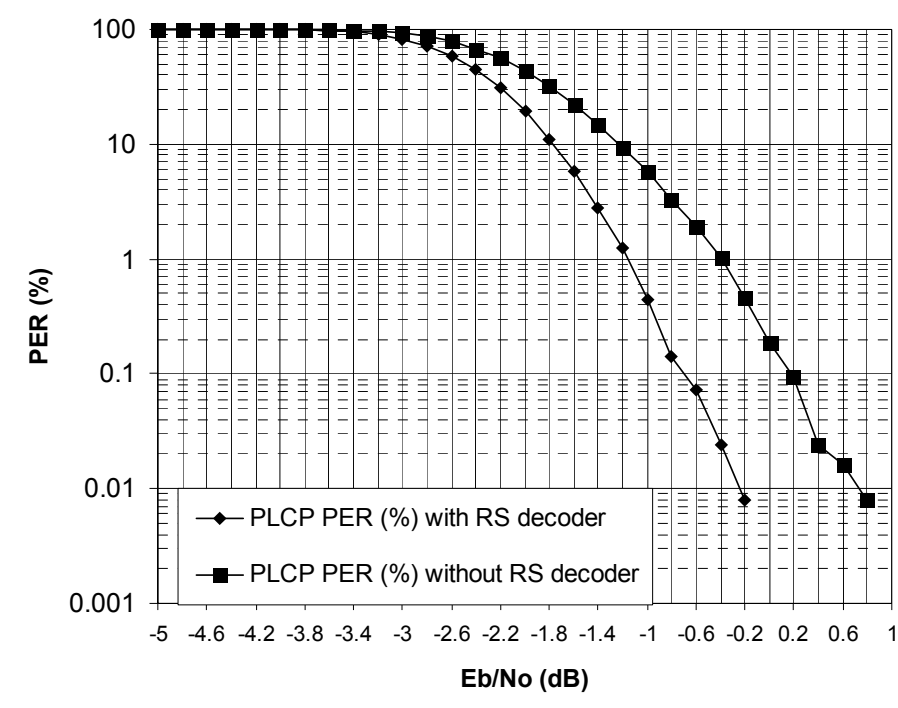

Fig. 2. PLCP Header Packet Error Rate (PER) (\%) using Channel Model 4 (CM4) with and without the RS Decoder present in the receiver.

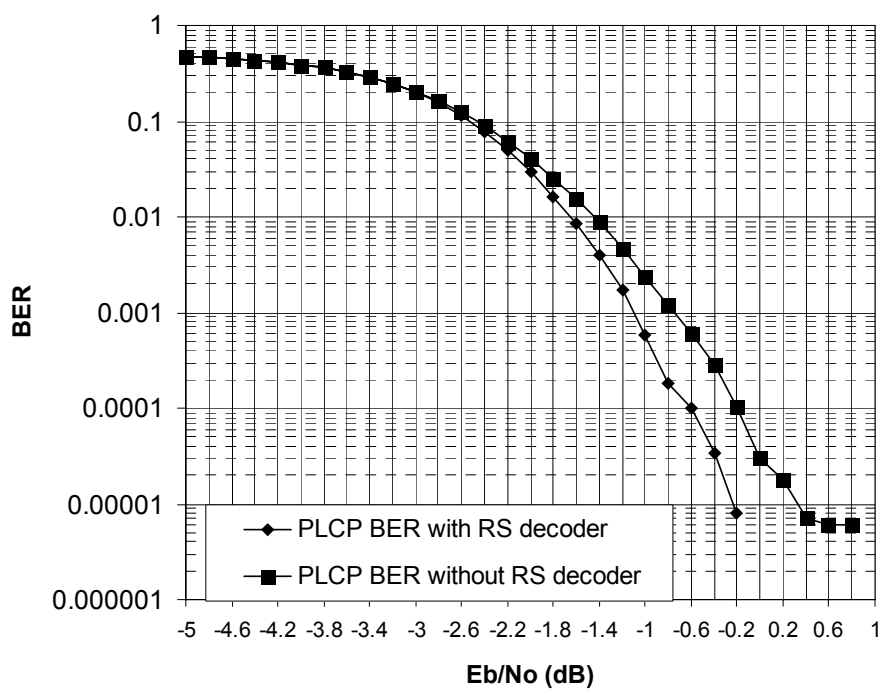

Fig. 3. PLCP Header Bit Error Rate (BER) using Channel Model 4 (CM4) with and without the RS Decoder present in the receiver.

the minimal receiver may be selected. If selected however, the performance of the spreading data rates is degraded to that of the non-spreading data rates with the same coding (e.g. the $200 \mathrm{Mbit} / \mathrm{sec}$ performance will be similar to the $400 \mathrm{Mbit} / \mathrm{sec}$ performance).

For the fast data rates (320 Mbit/sec rates and above) no spreading is used, but some diversity can be exploited in the DCM as presented above. This diversity feature may be exploited by various Maximum Ratio Combining (MRC) techniques. However, MRC algorithms can tend to be too complicated for the gain benefit in this application.

\section{E. Memory Requirements}

In this section the memory requirements are defined assuming that the PLCP Header and PSDU are encoded in sequential order, thus allowing intermediate memory re-usage. It is also assumed that the baseband timing is such that only 1 
encoded OFDM frame needs to be buffered in a queue ready for transmission. Table III presents the memory requirements.

In the transmitter, the MAC frame arrives over an interface to the PHY constituting the PSDU octets to be sent (maximum 4095) and the MAC TX_VECTOR parameters list (29 bits minimum and 10 octets for the MAC Header) giving a signaling buffer totaling 4105 octets. However, if the TX_VECTOR and PSDU vectors are separated then memory operations can be reduced. The PSDU and MAC FCS are sent LSB first; therefore in-place bit-reordering may be needed. Tail bits and pad bits need to be added at a maximum length of 148 octets, but serially a number of zero value bits can be pushed though the encoder requiring no extra memory. To create the PSDU all the bit operations before the interleaver may be done serially requiring no extra buffering, but an intermediate buffer of 1200 bits is required to hold each interleaver buffer. QPSK modulation can be done serially, but DCM requires an input buffer of 200 bits to operate on (as some indexes are changed). Both the QPSK and DCM require a maximum output buffer of 100 complex 6-bit values [14] and a 128 word 6-bit complex buffer (or 2 real value buffers) are required to construct the input to the IFFT, but it is possible to merge these buffers. The IFFT output will need to be buffered ready for transmission.

The memory requirements are generally similar for the receiver and in a transceiver design the memory may be shared, but note that the Convolutional decoder needs a lot of memory to hold soft decisions (in the case of a soft-bit decoder) before state-pinned trace-back of the whole PSDU. Also, buffering is needed to hold the spread symbol and to decode the spread symbol before a decision is made to merge the main and spread symbols. As can be seen from Table III, just fewer than $22 \mathrm{k}$ octets of algorithmic data memory are required by the transceiver baseband (assuming 1 OFDM buffering and a soft convolutional decoder).

\section{Conclusions}

This paper has presented a background of Multiband OFDM and its clear importance to Consumer Electronics. Design issues have been considered to give the designer more degrees-of-freedom in the specification of the physical transceiver so as to enable low cost, low power and small size physical digital baseband designs. Design performance vs. size/power tradeoffs and their performance have been shown for the RS decoder, memory and time domain spreading.

\section{REFERENCES}

[1] Federal Communications Commission, "New Public Safety Applications And Broadband Internet Access Among Uses Envisiged by FCC Authorization of Ultra-Wideband Technology", press release $14^{\text {th }}$ Feb 2002 ,

http://www.fcc.gov/Bureaus/Engineering_Technology/News_Releases/2 002/nret0203.html [Accessed 8th September 2006]

[2] J. Ellis, K. Siwiak and R. Roberts, "TG3a Technical Requirements", IEEE P802.15 Working Group for Wireless Personal Area Networks (WPANs), IEEE P802.15-03/030-SG3a, December 2002, http://grouper.ieee.org/groups/802/15/pub/2003/Jan03/03030r0P80215 TG3a-Technical-Requirements.doc [Accessed 8th September 2006]

[3] R. Fisher, et al, "DS-UWB Physical Layer Submission to 802.15 Task
Group 3a”, IEEE standard proposal P802.15-04/0137r3, July 2004, http://grouper.ieee.org/groups/802/15/pub/04/15-04-0137-03-003amerger2-proposal-ds-uwb-update.doc [Accessed 8th September 2006]

[4] A. Batra, et al, "Multi-band OFDM physical layer proposal for IEEE 802.15 task group 3a", IEEE standard proposal P802.15-03, March 2004 , http://grouper.ieee.org/groups/802/15/pub/2003/Jul03/03268r3P802 15 TG3a-Multi-band-CFP-Document.doc [Accessed 8th September 2006]

[5] Wireless-USB Implementers Forum http://www.usb.org/developers/wusb/ [Accessed 8th September 2006]

[6] USB Implementers forum, "Wireless Universal Serial Bus Specification, Revision 1.0", $12 \quad$ May 2005, http://www.usb.org/developers/wusb/wusb_2006_0302.zip [Accessed 8th September 2006]

[7] FCC, "ET Docket No. 04-352", $3^{\text {rd }}$ Sept 2004, http://hraunfoss.fcc.gov/edocs public/attachmatch/DOC-251750A1.doc [Accessed 8th September 2006]

[8] FCC, $10^{\text {th }}$ March 2005, http://hraunfoss.fcc.gov/edocs_public/attachmatch/DOC-257308A1.pdf [Accessed 8th September 2006]

[9] ECMA-368, "High Rate Ultra Wideband PHY and MAC Standard", December 2005, http://www.ecmainternational.org/publications/files/ECMA-ST/ECMA-368.pdf [Accessed 8th September 2006]

[10] Multiband OFDM, "MultiBand OFDM Physical Layer Proposal for IEEE 802.15.3a", September 2004, http://www.wimedia.org/imwp/idms/popups/pop_download.asp?Content $\underline{I D=6516}$ [Accessed 8th September 2006]

[11] ECMA-369, "MAC-PHY Interface for ECMA-368", December 2005, http://www.ecma-international.org/publications/files/ECMA-ST/ECMA369.pdf [Accessed 8th September 2006]

[12] http://www.theautochannel.com/news/2006/01/05/206018.html [Accessed 8th September 2006]

[13] J. Foerster, "Channel Modeling Sub-committee Report Final", IEEE P802.15 Working Group for Wireless Personal Area Networks (WPANs), IEEE P802.15-02/490-SG3a. $7^{\text {th }} \quad$ Feb. 2003 http://grouper.ieee.org/groups/802/15/pub/2003/Mar03/02490r1P80215 SG3a-Channel-Modeling-Subcommittee-Report-Final.zip [Accessed 8th September 2006].

[14] R. S. Sherratt and S. Makino, "Numerical Precision Requirements on the Multiband Ultra-Wideband System for Practical Consumer Electronic Devices", IEEE Transactions on Consumer Electronics, Volume 51(2), May 2005, pp. 368-392.

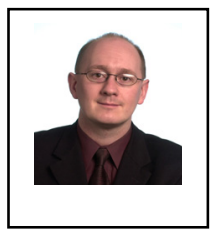

R. Simon Sherratt (M'97-SM'02) was born in Heswall, UK. He received his B.Eng. degree in Electronic Systems and Control Engineering from Sheffield City Polytechnic UK in 1992, his M.Sc. degree in data telecommunications from the University of Salford UK in 1994 and his Ph.D. degree in video signal processing also from the University of Salford in 1996. Since 1996, he has been a Lecturer in Electronic Engineering at the University of Reading where he is now a senior lecturer in consumer electronics and currently head of Electronic Engineering. His research topic is signal processing in consumer electronic devices concentrating on equalization, communications layer 1, DSP architectures and adaptive signal processing. Eur Ing Dr. Sherratt is a senior member of the IEEE, member of the IEEE Consumer Electronics Society AdCom (2003-2005, 2006-2008) holding the IEEE Consumer Electronics Society International Symposium on Consumer Electronics liaison officer post (2005-) and the Society awards chair post (2006-), member of the IEEE Transactions on Consumer Electronics publications committee (2004-), IEEE International Conference on Consumer Electronics ExCom and vice-technical chair (2006), chair of the IEEE International Symposium on Consumer Electronics 2004 and committee member for 2002, 2003, 2005 and 2006, founder and current chair of the IEEE UKRI Consumer Electronics and Broadcast Technology Joint Chapter. He received the IEEE Chester Sall $1^{\text {st }}$ place best Transactions paper award in 2004 and the best paper in the IEEE International Symposium on Consumer Electronics 2006. He received the IEEE Consumer Electronic Society International Chapters Outstanding Service Award in 2005. 